\title{
CORRECTION
}

\section{Correction to: Experimental analysis of transonic buffet on a 3D swept wing using fast-response pressure-sensitive paint}

\author{
Yosuke Sugioka $^{1} \cdot$ Shunsuke Koike $^{2} \cdot$ Kazuyuki Nakakita $^{2} \cdot$ Daiju Numata $^{1} \cdot$ Taku Nonomura $^{1} \cdot$ Keisuke Asai $^{1}$
}

Published online: 3 June 2020

○) Springer-Verlag GmbH Germany, part of Springer Nature 2020

\section{Correction to: Experiments in Fluids (2018) 59:108 https://doi.org/10.1007/s00348-018-2565-5}

There are typographical errors in the original publication of this article. We apologize for these mistakes.

The first error is the sentence in the fourth paragraph on page 2. The correct sentence is "The bump Strouhal number, which characterizes $3 \mathrm{D}$ buffet $\left[\mathrm{St}=O\left(10^{-1}\right)\right]$, is much higher than the shock oscillation frequency of $2 \mathrm{D}$ buffet $\left[\mathrm{St}=O\left(10^{-2}\right)\right]$."

The second error is the equation on page 17 in the left column. The correct equation is: $\lambda=U_{C} f$.

Note that the values shown in the original publication were calculated according to the correct equation. Hence, the results and conclusions are not affected by the present erratum.

Publisher's Note Springer Nature remains neutral with regard to jurisdictional claims in published maps and institutional affiliations.
The original article can be found online at https://doi.org/10.1007/ s00348-018-2565-5.

Yosuke Sugioka

sugioka.yousuke@aero.mech.tohoku.ac.jp

1 Department of Aerospace Engineering, Graduate School of Engineering, Tohoku University, 6-6-01 Aramaki-Aza-Aoba, Aoba-ku, Sendai, Miyagi 980-8579, Japan

2 Aeronautical Technology Directorate, Japan Aerospace Exploration Agency, Chofu, Tokyo, Japan 